\title{
Improved support vector machine using optimization techniques for an aerobic granular sludge
}

\author{
Nur Sakinah Ahmad Yasmin ${ }^{1}$, Norhaliza Abdul Wahab ${ }^{2}$, Aznah Nor Anuar ${ }^{3}$ \\ ${ }^{1,2}$ School of Electrical Engineering, Faculty of Engineering, Universiti Teknologi Malaysia, Malaysia \\ ${ }^{3}$ Department of Environment and Green Technology, Malaysia-Japan International Institute of Technology, Malaysia
}

\section{Article Info \\ Article history: \\ Received Dec 18, 2019 \\ Revised Mar 7, 2020 \\ Accepted Apr 13, 2020}

\section{Keywords:}

Aerobic granular sludge Modelling

Support vector machine

SVM-GA

SVM-PSO

\begin{abstract}
Aerobic granular sludge (AGS) is one of the treatment methods often used in wastewater systems. The dynamic behavior of AGS is complex and hard to predict especially when it comes to a limited data set. Theoretically, support vector machine (SVM) is a good prediction tool in handling limited data set. In this paper, an improved SVM using optimization approaches for better predictions is proposed. Two different types of optimization are built which are particle swarm optimization (PSO) and genetic algorithm (GA). The prediction of the models using SVM-PSO, SVM-GA and SVM-Grid Search are developed and compared prior to several feature analysis for verification purposes. The experimental data under hot temperature of $50^{\circ} \mathrm{C}$ obtained from sequencing batch reactor is used. From simulation results, the proposed SVM with optimizations improve the prediction of chemical oxygen demand compared to the conventional grid search method and hence provide better prediction of effluent quality using AGS wastewater treatment systems.
\end{abstract}

This is an open access article under the CC BY-SA license.

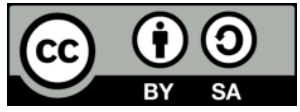

Corresponding Author:

Norhaliza Abdul Wahab,

School of Electrical Engineering, Faculty of Engineering,

Universiti Teknologi Malaysia, 81310 Skudai Johor, Malaysia.

Email: aliza@fke.utm.my

\section{INTRODUCTION}

Wastewater treatment is a process of converting wastewater into an effluent before it can be safely returned to the water cycle. It is full of contaminants including toxins, bacteria and harmful chemicals that are not safe to be consume. The main goals of wastewater treatment is to eliminate all the contaminants and ensure the water is safe to discharge back to the water cycle. As the demands of urban area grows rapidly, the wastewater treatment nowadays has various kind of water treatment ranging from low price to highly cost operations. Compared with drawbacks of the system such as required large space area for separation aeration and settling tanks, produced low biomass concentration in aeration tank but high energy consumption and operates in space rather than time of conventional activated sludge system, a new technology using sequencing batch reactor (SBR) is developed.

New technology which relies on the growth of aerobic granular sludge (AGS) can helps to overcome the limitation of conventional activated sludge system. It has been proved that the formation of AGS has a short settling time when inoculated with activated sludge and operate using SBR [1]. Since then, AGS technology has been attracting attention among the researcher for its great potential in reducing footprints while increasing the efficiency of wastewater treatment plants. AGS technology has been one of the promising technologies in biological treatment. Some of the advantages of AGS technology are has a compact and regular structure of granules make AGS able to withstand the high load of toxic compound, high biomass retention and have a good settle ability [2]. This process of aerobic granulation can be seen 
happened in SBR. Many studies have extensively investigated the fundamental of AGS in SBR [3-5]. A study to identify the most vital feature that influencing the development of AGS process for instance settling time, loading rate, hydrodynamic shear force and substrate composition [6] is demonstrated in a lab scale.

The formation of AGS is a complex biological process and it influenced by other parameters that need to be further examined. Generally the study of AGS using SBR system was performed at $20^{\circ} \mathrm{C}$ until $25^{\circ} \mathrm{C}$ or lower $[6,7]$. Hence, it may not suitable for the country with high climate weather temperature such as Saudi Arabia. Further investigation is needed to study the effect on AGS process at three different high temperature which are $30^{\circ} \mathrm{C}, 40^{\circ} \mathrm{C}$ and $50^{\circ} \mathrm{C}$ by using SBR system. However, AGS is a very complex occurrence of microbial immobilization [8]. There are numerous internal interaction among the process between the variables such as growth and sludge characteristic including oxygen transfer, biomass detachment and diffusion. Thus, a comprehensive mathematical model that can takes all these parameters into consideration is needed in order to establish a good model. As a highly approach for the limited data set, Support Vector Machine has been proposed to develop the model [9]. Many field of studies has been applied SVM such as in prediction [10], pattern recognition problem [11], classification [12], regression [13, 14] and time series $[12,15]$. In addition, SVM has been reported outperformed traditional statistical learning method such as artificial neural network. Thus, more studies are focusing on this SVM technique as SVM has been arousing more.

In wastewater treatment, SVM was utilized widely in modelling process. The example of implementation of SVM technique on limited dataset can be found in [16], which SVM model is developed in order to study the relationship between membrane bioreactor and the affecting factors of fouling. This study reported that SVM technique can assure a good prediction results compared to neural network model since the depending of the training dataset for SVM technique is lesser than neural network. In wastewater treatment, the same method was observed, [17] implement SVM technique to model effluent quality with cyclic activated sludge process. This study reported to gives high prediction accuracy with small learning ability and good generalization. Other than that, [18] presented a successful SVM technique that was applied to predict the permeate flux and rejection of bovine serum albumin.

The results shows SVM outperformed artificial neural network (ANN) due to ANN technique needs to rely on the one experience and expertise while SVM technique is rely on statistical theory. The implementation by [19] in predicting the different sample size of membrane permeate flux dead end microfiltration of activated sludge reactor also shows SVM model outperformed as compared to back propagation neural network. This study shows that small sample size of SVM yields higher accuracy. This is because of the rigorous foundation of mathematical and theoretical of SVM despite needs to rely on the designer knowledge itself.

In SVM algorithm, two parameters are involved which are cost, $C$ and gamma, $\gamma$. It important to select best pair of these two parameters as it will affect the performance of the results. Common method used usually are trial and error method or by using grid search. However, these types of method is a conventional way and its time consuming [20]. Hence, this paper implement an optimization of a SVM model by using genetic algorithm (GA) and particle swarm optimization (PSO). The main goal of this paper is to model and optimize the SVM model by using limited dataset. The fitness function used in this work is to minimize the mean square error. In this study, the 60 days data which contains of 21 samples under the temperature of $50^{\circ} \mathrm{C}$ is utilized to develop the model of AGS by using SBR systems. As for this paper, it is consist of five main sections and are organized as follows. The first section started with the background of this study and several literature on the proposed previous method. Then, second section will be presented modelling of SVM with the proposed optimization method which are GA and PSO. Third section is the detailed of the experimental setup of this study while in the fourth section is the results and discussion. Finally, the fifth section gives conclusion and future works based on this study.

\section{RESEARCH METHOD}

SVM was first introduced by Vapnik (1995) [13]. The idea of SVM is based on structural risk management. Basically, SVM is a supervised learning method that is capable in generating predictive outputs of the model and was used for classifying unseen patterns into their categories. Recently, SVM technique has been extended its function in solving function estimation and regression problems. The SVM technique is based on the concept of optimal hyper lane that separates sample of two different classes by considering the widest gap between them. SVM technique solves a quadratic problem in which the objective function in obtained by combination of regularization term and loss function. Support vector regression basically is an extension of SVM regression. The main purpose of SVM regression is to find a function that has a most 
$\gamma$ deviation from the actually obtained objectives for all training dataset and at the same time it would be as flat as possible.

\subsection{Support vector machine}

The general theory of SVM regression function is expressed as follows in (1).

$$
y=f(x)=\omega \cdot x+b
$$

where scalar output is denoted as $y$, weight vector is denoted as $\boldsymbol{\omega}$, while vector and bias is denoted as $x$ and $b$, respectively. These coefficients are estimated by introducing slack variables, $\xi_{i}$ and $\xi_{i}^{*}$, to minimizing the regularized risk function. These variables represent the distance from the actual values to the corresponding boundary value of $\mathcal{E}$-tube. The SVM regression function can be expressed by (2) and (3).

$$
\text { Minimize } \quad \phi(\boldsymbol{\omega})=\frac{1}{2}\|\boldsymbol{\omega}\|^{2}-C \sum_{i=1}^{n}\left(\xi_{i}-\xi_{i}^{*}\right) \cdot C \geq 0
$$

where

$$
\text { Subject to }\left\{\begin{array}{c}
y-\boldsymbol{w} \boldsymbol{x}-b \leq \varepsilon+\xi_{i}, \\
\boldsymbol{w} \boldsymbol{x}-b-y \leq \varepsilon+\xi_{i}^{*}, i=1,2, \ldots, \\
\xi_{i}, \xi_{i}^{*} \geq 0,
\end{array}\right.
$$

The constant $C$ is the cost factor and determines the trade of between approximation error and weight factor. $\gamma$ is the radius of the tube within where the regression function must lie. Different kernel function have been used in SVM regression problems such as linear kernel, polynomial kernel and Gaussian kernel. Each type of kernel function has its own parameters which will affect the flexibility of regression function. Radial basis function (RBF) is one of those popular kernel function in SVM that has been widely used in different types of studies. When there is no prior knowledge about data characteristics, RBF kernel is recommended as RBF kernel is intent to give good performance under smoothness assumption. After calculating $\alpha_{i}^{+}$and $\alpha_{i}^{=}$, thus, the new generated predicted output of SVM regression function for each $x^{\prime}$ is can be given as (4) [21].

$$
y^{\prime}=\sum_{i=1}^{L}\left(\alpha_{i}^{+}-\alpha_{i}^{=}\right) x_{i} \cdot x^{\prime}+b
$$

\subsection{Genetic algorithm}

GA is based on evolutionary algorithms. The basic concept of GAs is designed to simulate processes in natural systems especially for evolution. These algorithms are based on survival principle of the fittest member in population which tries to retain genetic information from generation to generation [22]. One of the GAs advantage characteristics is their inherent parallelism regarding others algorithm that are serial. It would eliminated one path turns out to be dead end and other promising paths will be continued. Other significant capability of GAs is in finding global optimum among many local optima even on the condition of complex problems [23].

\subsection{Particle swarm optimization}

Basically, PSO is similar to GA since PSO itself is an evolutionary computation technique. PSO is based on population searching technique that utilize the idea of social sharing informations. In PSO, particles are flown through hyper dimensional search space. The changes of the particles position are based on social psychological tendency which are the particles are following the success of other particles. Thus, the changes of a particles within the swarm are influence by experience and knowledge of its neighbour. Consequently, the behaviour of particles is also affected by that of other particles within the swarm. This results in the prediction of a model since that the social behaviour of the particles stochastically return toward previously successful areas in the search space. The process of PSO and GA optimization are describe in the flow chart in Figure 1. 


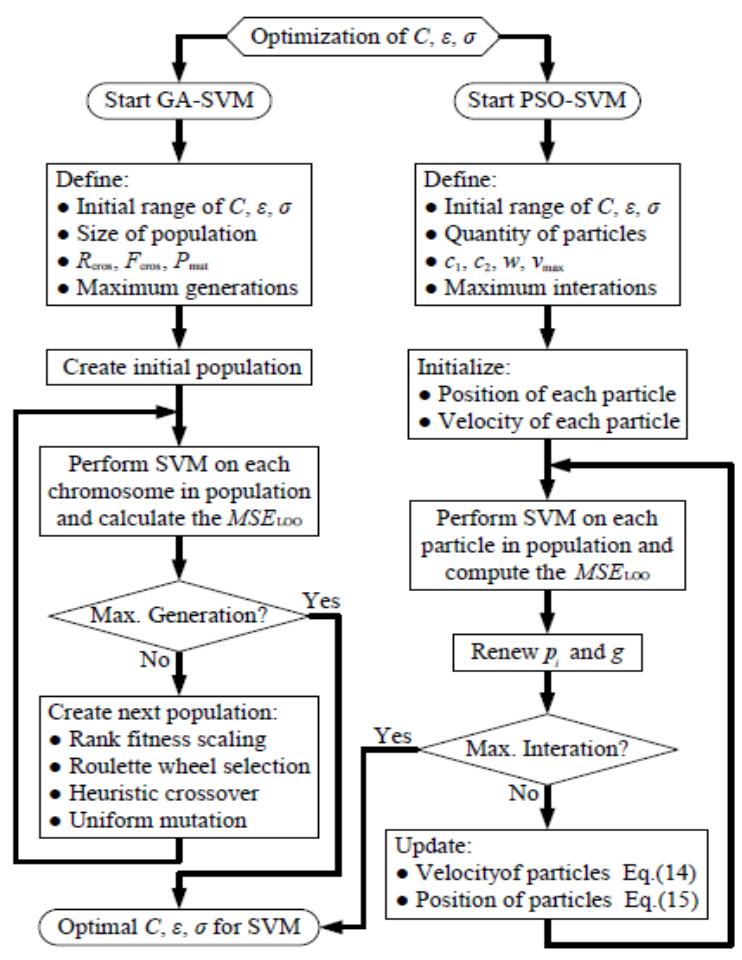

Figure 1. SVM-PSO/GA procedure

\section{EXPERIMENT SETUP}

In this study, the experimental setup was carried out in the double-walled cylindrical column of SBR. The height and internal diameter of SBR are $100 \mathrm{~cm}$ and $6.5 \mathrm{~cm}$, respectively. All the operation such as influent reading, effluent discharge and aerator pump was controlled by programmable logic controller system with the setting time for each phase. The working temperature of SBR were controlled using water bath sleeves and thermostat at three different temperatures which are $30^{\circ} \mathrm{C}, 40^{\circ} \mathrm{C}$ and $50^{\circ} \mathrm{C} \pm 1{ }^{\circ} \mathrm{C}$. In this study, the AGS bioreactor operated at $50^{\circ} \mathrm{C} \pm 1^{\circ} \mathrm{C}$ is considered. The influent parameters of AGS bioreactor that used as input of the model are chemical oxygen demand (COD), total phosphorus (TP), total nitrogen $(\mathrm{TN})$, ammonia nitrogen (AN) and mixed liquor suspended solid (MLSS). The output parameter of the model is the effluent COD. Figure 2 shows the schematic diagram of the AGS of SBR system.

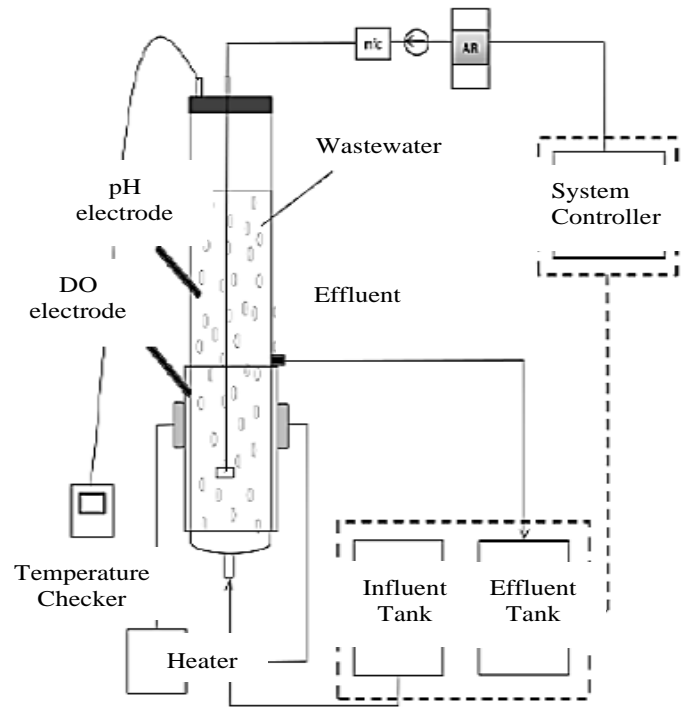

Figure 2. Schematic diagram of laboratory scale AGS bioreactor 


\section{RESULT AND DISCUSSION}

In this section, the modelling and optimization results of AGS are presented. As mention in Section 3 , only bioreactor of $50^{\circ} \mathrm{C} \pm 1{ }^{\circ} \mathrm{C}$ is considered. The collection of the real experimental data is limited to only 21 dataset. Hence, the data was split using 10-fold cross validation (CV) as it was suggested for limited dataset [24]. The first 53\% from the experimental data was used as the training dataset while the remaining $47 \%$ is used as testing dataset. The 10-CV is then combined with grid search method in order to choose the pair of parameters of $C$ and $\gamma$. The $\mathrm{CV}$ operated by using 9-fold from 53\% of training dataset to train and leave one last fold as a test dataset. The training model was repeated for ten times and the average performance are calculated. Then it will tested again on the $47 \%$ to verify the perfomance. The results are measured using root mean square error (RMSE) performance criteria.

\subsection{Validation and evaluation of the SVM-GA model}

In statistic, it is important to check the validity of the regression model. The validation of the SVM regression model involve by analysing the goodness fit of the model, checking the residual plots plot are random and by checking the performance of prediction model towards unknown data (testing data) if the performance deteriorates substantially when applied. In order to accurately and objectively map the precision of the model, the prediction result of the effluent COD concentration are validated based on statistical parameter such as highest correlation coefficient $\left(\mathrm{R}^{2}\right)$, and lower mean square error (MSE) values. The mathematical formulas are given as (5) and (6) [25].

$$
R^{2}=1-\frac{\sum_{i=1}^{n} w_{i}\left(y_{i}-\hat{y}_{i}\right)^{2}}{\sum_{i=1}^{n} w_{i}\left(y_{i}-\bar{y}\right)^{2}}
$$

Where $\hat{y}_{i}$ denoted as prediction data, $\bar{y}$, as the mean of actual data

$$
M S E=\frac{\sum_{i=1}^{n} w_{i}\left(y_{i}-\hat{y}_{i}\right)^{2}}{n-m}
$$

Where $y_{i}$, as actual value from the measurement data, $n$ is the number of responses minus the number of fitted coefficients $m$ estimated from the response value. Figure 3 (a), Figure 4 (a) and Figure 5 (a) present the cross plot between the measured and predicted results for SVM PSO, SVM-GA and SVM Grid-search during testing output, respectively. The plots have obtained coefficients values of 94.59\%, 94.11\% and $94.65 \%$ during testing phase. These values demonstrate high accuracy of each models as it is closed to unity. Although the high coefficient indicate a good model, but there is a need to confirm stochastic nature of any intrinsic errors. Hence, residuals is plotted for this purpose. As shown in Figure 3 (b), Figure 4 (b) and Figure 5 (b) the residuals are evenly dispersed around zero line ( $\mathrm{x}$-axis).

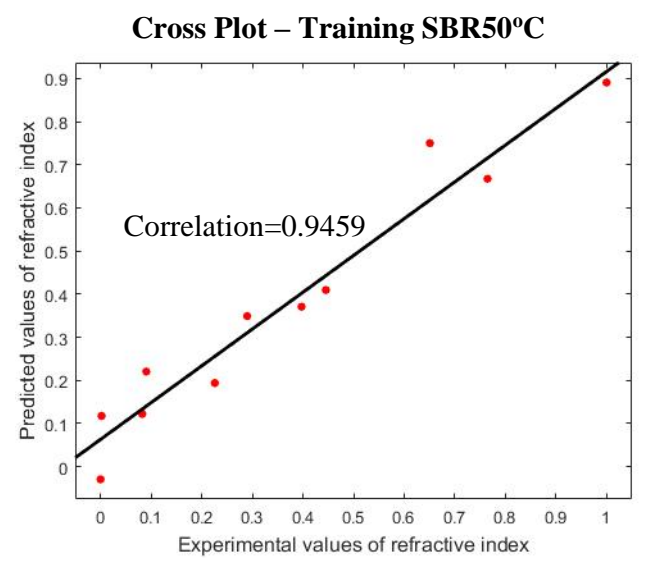

(a)

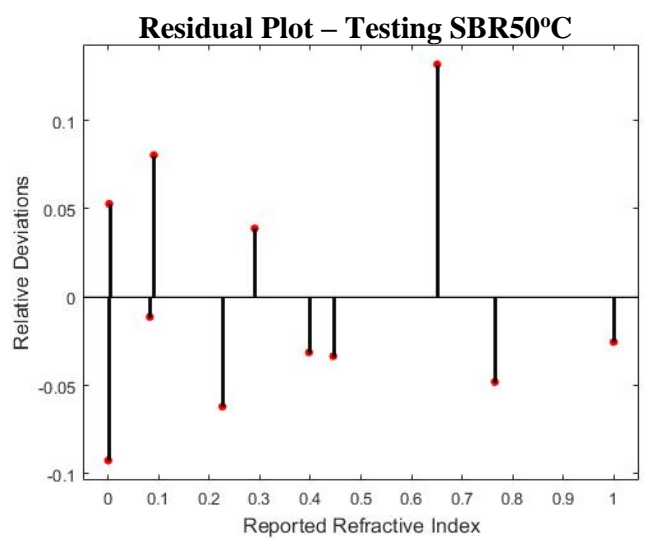

(b)

Figure 3. (a) Cross plot of SVM-PSO during testing phase for SBR50oC, (b) Residuals plot of the SVM-PSO model's relative error deviations during testing phase for $\mathrm{SBR} 50^{\circ} \mathrm{C}$ 


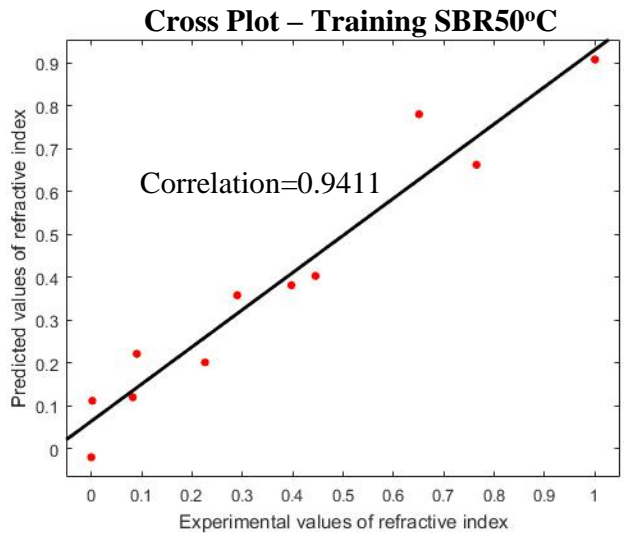

(a)

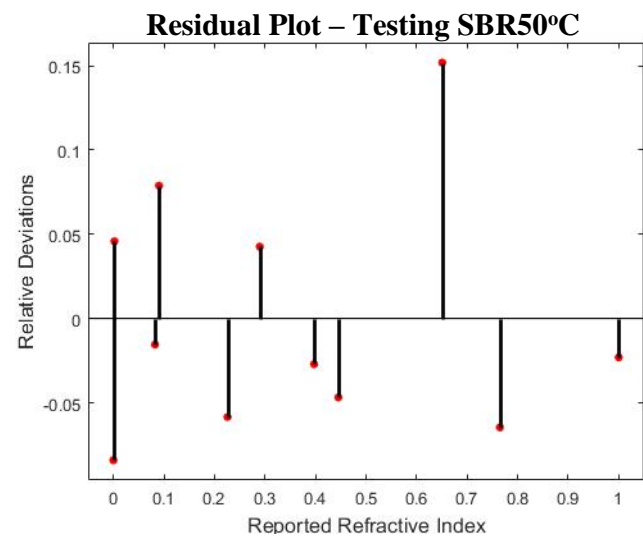

(b)

Figure 4. (a) Cross plot of SVM-GA during testing phase for SBR50 ${ }^{\circ} \mathrm{C}$, (b) Residuals plot of the SVM-GA model's relative error deviations during testing phase for SBR $50^{\circ} \mathrm{C}$

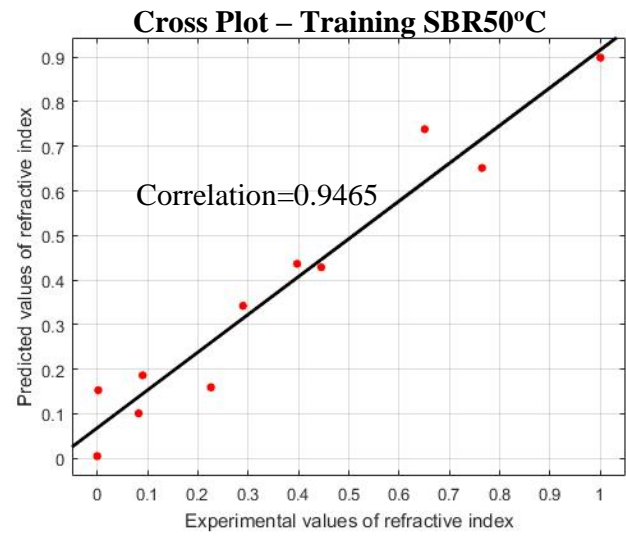

(a)

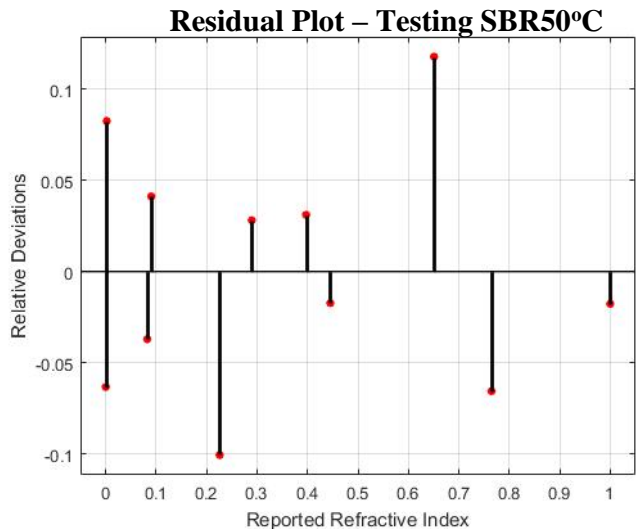

(b)

Figure 5. (a) Cross plot of SVM-Grid Search during testing phase for SBR50 ${ }^{\circ} \mathrm{C}$, (b) Residuals plot of the SVM-Grid search model's relative error deviations during testing phase for $\mathrm{SBR} 50^{\circ} \mathrm{C}$

The RMSE values obtained are $0.07123,0.07569$ and 0.0704 , respectively which closed to zero. Hence, these results are the indicative of high performing model that validate the generalizability and accuracy of the models achieved. The overall summarized results of the cross and residuals plot of SVM-PSO, SVM-GA and SVM-Grid Search are shown in Table 1.

Table 1. Statistical performance for SVM-PSO, SVM-GA and SVM-Grid search model

\begin{tabular}{ccccccc}
\hline Statistical Parameters & \multicolumn{2}{c}{ SVM-PSO } & \multicolumn{2}{c}{ SVM-GA } & \multicolumn{2}{c}{ SVM-Grid Search } \\
\cline { 2 - 7 } SBR50 ${ }^{\circ} \mathrm{C}$ & Training & Testing & Training & Testing & Training & Testing \\
\hline SSE & 0.01781 & 0.04567 & 0.01538 & 0.05156 & 0.02417 & 0.04463 \\
R $^{2}$ & 0.9783 & 0.9459 & 0.9819 & 0.9411 & 0.9715 & 0.9465 \\
Adjusted R & 0.9755 & 0.9399 & 0.9796 & 0.9345 & 0.9680 & 0.9405 \\
RMSE & 0.04719 & 0.07123 & 0.04384 & 0.07569 & 0.05497 & 0.0704 \\
P1 & 0.8593 & 0.8529 & 0.8764 & 0.8665 & 0.8722 & 0.8481 \\
P2 & 0.04454 & 0.06321 & 0.04409 & 0.06449 & 0.0416 & 0.06843 \\
\hline
\end{tabular}

\subsection{Development of SVM grid search model}

Generally, common method usually used in determine the parameters used in SVM are using trial and error or grid search method. To make a clear comparison with optimization method which are PSO and GA, grid search algorithm is developed. From the tuning parameters grid search for cost and gamma values, the algorithm found the local minima with minimum error RMSE of 0.0128. The pairs values of cost and gamma obtained are 4 and 0.125 . By using the values of parameters obtained, a graph for training 
and testing data are plotted as shown in Figure 6. From the plotted graph, it shows that the prediction of SVM-Grid search during training model gives good correlation with low MSE value which are 96.17\% and 0.0041 , respectively. To validate the results, the algorithm is then used on testing data (unknown data) and the graph obtained the values $\mathrm{R}^{2}$ of $93.43 \%$ and MSE of 0.0065 .
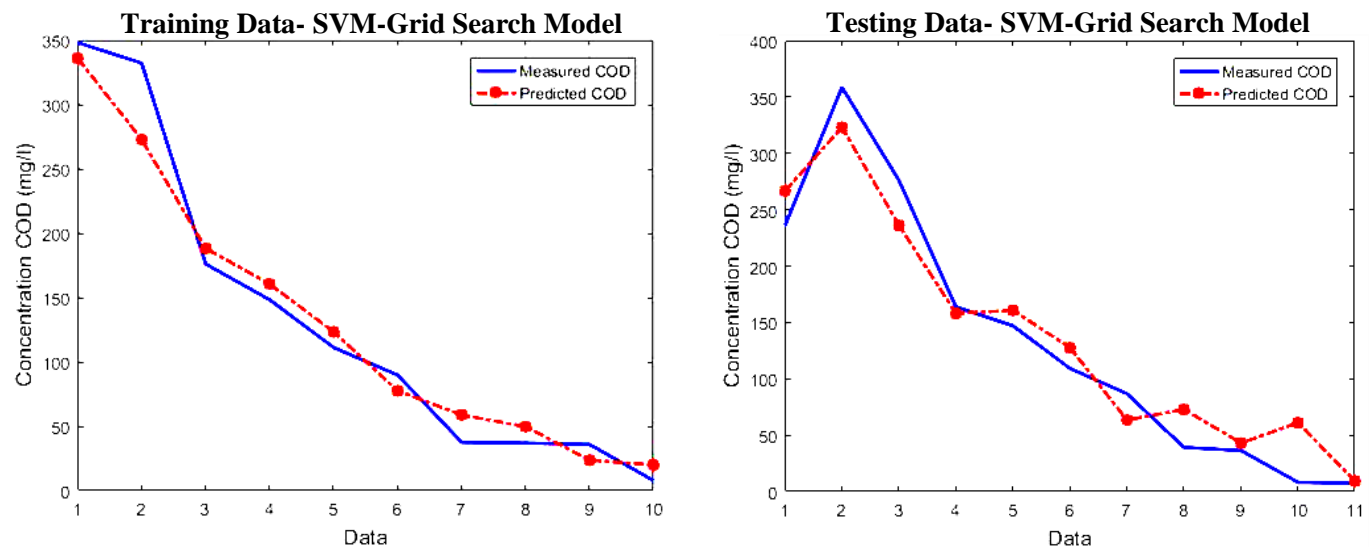

Figure 6. Comparison between measured and predicted of SVM Grid-Search for training and testing data

\subsection{Development of hybrid SVM-PSO and SVM-GA model}

The development of optimization method using PSO and GA are developed using Matlab algorithm. Both technique shows good agreement accuracy in predicting the model. The simulation results of fitness curve for SVM-PSO and SVM-GA are measured using MSE performance criteria. The closer the value to the zero is better for the prediction model. From the iteration, the system found the best local minima for SVM-PSO with the fitness value of 0.0062. The results of fitness curve for SVM-GA obtained MSE value of 0.0065 . The parameters of cost and gamma gives by SVM-PSO are 3.0522 and 0.2471 . As for SVM-GA, the value of parameters achieved are 2.0638 and 0.3318 for cost and gamma. This shows that the fitness values obtained from SVM-PSO is slightly better as compared to SVM-GA.

Figure 7 shows the comparison graph between SVM-PSO, SVM-GA and SVM-Grid search using the parameters obtained earlier. From the graph during training phase, it shows that SVM-GA obtained the highest correlation coefficient followed by SVM-PSO and SVM-Grid search. The values of correlation coefficient obtained are $98.19 \%, 97.83 \%$ and $96.17 \%$ with the corresponding MSE values of $0.0032,0.0040$ and 0.0041 respectively. From the result, it can be seen that all three method achieved a very well prediction as it is higher than $90 \%$ in predicting concentration COD effluent. Meanwhile during testing phase, the testing results shows SVM-PSO achieved the best prediction result with correlation coefficient value of $94.59 \%$ and MSE value of 0.0064 . It followed by SVM-GA with $\mathrm{R}^{2}$ of $94.11 \%$ and MSE value of 0.0067 . SVM-Grid search obtained the lowest prediction as the values achieved are $93.43 \%$ and 0.0070 for $\mathrm{R}^{2}$ and MSE.
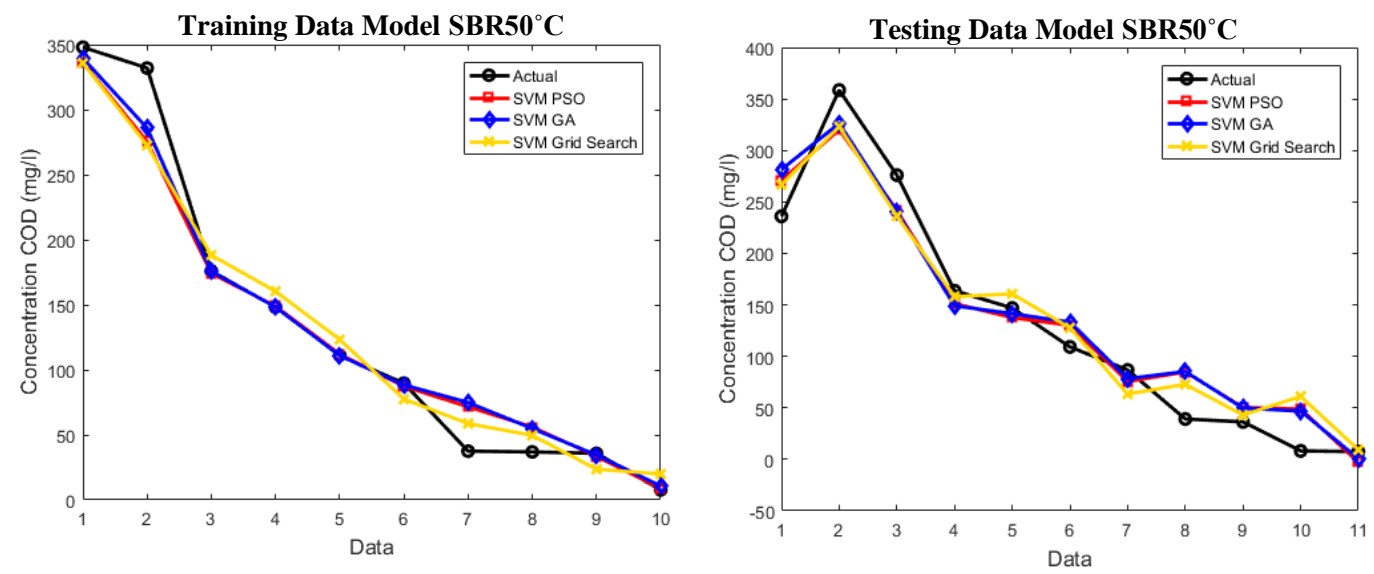

Figure 7. Comparison graph between measured and predicted of SVM-PSO, SVM-GA and SVM-grid search for training and testing data 
The overall performance comparison for training and testing results is shown in Table 2. From the results obtained, it clearly shown that with the optimization method could enhance the results instead of using conventional way of grid search method. The hybridization of SVM-PSO achieved the highest performance during testing dataset followed by SVM-GA and SVM-grid search.

Table 2. Overall performance for SVM-PSO, SVM-GA and SVM-Grid search model

\begin{tabular}{|c|c|c|c|c|c|c|c|}
\hline \multirow{2}{*}{ SBR } & \multirow{2}{*}{ Model } & \multirow{2}{*}{$C$} & \multirow{2}{*}{$\gamma$} & \multicolumn{2}{|c|}{ Training } & \multicolumn{2}{|c|}{ Testing } \\
\hline & & & & $\mathrm{R}^{2}(\%)$ & MSE & $\mathrm{R}^{2}(\%)$ & MSE \\
\hline \multirow[t]{2}{*}{$50^{\circ} \mathrm{C}$} & SVM-PSO & 3.0522 & 0.2471 & 97.83 & 0.0040 & 97.83 & 0.0040 \\
\hline & SVM-GA & 2.0638 & 0.3318 & 98.19 & 0.0032 & 98.19 & 0.0032 \\
\hline
\end{tabular}

\section{CONCLUSION}

This paper presents the modelling and optimization techniques for AGS using SVM hybrid with PSO, GA and grid search. The SVM computational approach has been widely considered by a researchers due to its great ability in finding the local minimum, solved a non-linearity problems, and can be applied in high dimension system. This can be proven from the simulation results, it can be observed that the testing result using optimization method such as PSO and GA could produces better accuracy of the model prediction at the temperature of $50^{\circ} \mathrm{C}$. As compared to conventional method which is grid search, it is time-consuming are not able to obtained the best local minima in determine the parameters. This provide and evident that SVM-PSO and SVM-GA can give better prediction compared to SVM-Grid search model for limited training data. Basically, three major advantages of SVM computational approach can be highlight through this research. First, only three type of kernel parameters needed to be determined which are kernel function, cost of penalty and gamma value. Second, SVM itself are unique, optimal and global in solving quadratic problem. Third, SVM is well known with good generalization performance due to the implementation of SRM principle. For the recommendation of the future work, to improve the computational time of searching the SVM parameters in future, external optimization such as gravitational search algorithm (GSA) can be used as it was reported to give faster convergent and reliable to meet the objective function in many types of application. Hence, in SVM, GSA is a very potential optimization that can be considered.

\section{ACKNOWLEDGEMENTS}

The authors would like to thank the Ministry of Education (MOE) PRGS vote R.J130000.7851.4L702 for their financial support of this project and the Universiti Teknologi Malaysia UTMHIR vote $08 \mathrm{G} 74$ for supporting this work.

\section{REFERENCES}

[1] Y. Nancharaiah and G. K. K. Reddy, "Aerobic granular sludge technology: mechanisms of granulation and biotechnological applications,” Bioresource technology, vol. 247, pp. 1128-1143, January 2018.

[2] K.-Z. Su and H.-Q. Yu, "Formation and characterization of aerobic granules in a sequencing batch reactor treating soybean-processing wastewater," Environmental Science \& Technology, vol. 39, no. 8, pp. 2818-2827, 2005.

[3] L. De Bruin, H. Van der Roest, M. De Kreuk, M. Van Loosdrecht, and C. Uijterlinde, "Promising results pilot research aerobic granular sludge technology at WWTP Ede," Aerobic Granular Sludge, IWA Publishing, vol. 135142, 2005.

[4] Y.-Q. Liu, B. Moy, Y.-H. Kong, and J.-H. Tay, "Formation, physical characteristics and microbial community structure of aerobic granules in a pilot-scale sequencing batch reactor for real wastewater treatment," Enzyme and Microbial Technology, vol. 46, no. 6, pp. 520-525, May 2010.

[5] B.-J. Ni, W.-M. Xie, S.-G. Liu, H.-Q. Yu, Y.-Z. Wang, G. Wang, et al., "Granulation of activated sludge in a pilot-scale sequencing batch reactor for the treatment of low-strength municipal wastewater," Water Research, vol. 43, no. 3, pp. 751-761, February 2009.

[6] S. Lochmatter and C. Holliger, "Optimization of operation conditions for the startup of aerobic granular sludge reactors biologically removing carbon, nitrogen, and phosphorous," Water research, vol. 59, pp. 58-70, 2014.

[7] M. H. Ab Halim, et al., "Aerobic sludge granulation at high temperatures for domestic wastewater treatment," Bioresource technology, vol. 185, pp. 445-449, June 2015.

[8] J. B. Xavier, M. K. De Kreuk, C. Picioreanu, and M. C. van Loosdrecht, "Multi-scale individual-based model of microbial and bioconversion dynamics in aerobic granular sludge," Environmental science \& technology, vol. 41, no. 18, pp. 6410-6417, October 2007.

[9] N. S. A. Yasmin, N. A. Wahab, A. N. Anuar, and M. Bob, "Performance comparison of SVM and ANN for aerobic granular sludge,” Bulletin of Electrical Engineering and Informatics, vol. 8, no. 4, pp. 1392-1401, 2019. 
[10] V. Vapnik, "The nature of statistical learning theory," Springer-Verlag New York, 2013.

[11] Zhen-Yuan Jia, Jian-Wei Ma, Fu-Ji Wang, Wei Liu, "Hybrid of simulated annealing and SVM for hydraulic valve characteristics prediction," Expert Systems with Applications, vol. 38, no. 7, pp. 8030-8036, July 2011.

[12] B. Scholkopf et al., "Comparing support vector machines with Gaussian kernels to radial basis function classifiers," in IEEE Transactions on Signal Processing, vol. 45, no. 11, pp. 2758-2765, 1997

[13] V. Vapnik, S. E. Golowich, and A. Smola, "Support vector method for function approximation, regression estimation, and signal processing," Advances in neural information processing systems, pp. 281-287, 1997.

[14] Christopher J.C. Burges, Bernhard Schölkopf and Alexander J. Smola, "Advances in Kernel Methods-Support Vector Learning," MIT Press, Cambridge, UK, 1999.

[15] U. Thissen, R. Van Brakel, A. De Weijer, W. Melssen, and L. Buydens, "Using support vector machines for time series prediction," Chemometrics and intelligent laboratory systems, vol. 69, no. 1-2, pp. 35-49, 23 November 2003.

[16] N. S. A. Yasmin, N. A. Wahab, and Z. Yusuf, "Modeling of Membrane Bioreactor of Wastewater Treatment Using Support Vector Machine," in Asian Simulation Conference 2017, pp. 485-495, 2017.

[17] W. Li-juan and C. Chao-bo, "Support Vector Machine Applying in the Prediction of Effluent Quality of Sewage Treatment Plant with Cyclic Activated Sludge System Process," 2008 IEEE International Symposium on Knowledge Acquisition and Modeling Workshop, Wuhan, pp. 647-650, 2008.

[18] X. Xi, Z. Wang, J. Zhang, Y. Zhou, N. Chen, L. Shi, et al., "Prediction of impacts of fabrication conditions on the filtration performance of homemade VC-co-VAc-OH microfiltration membrane by Support Vector Machine (SVM)," Desalination and Water Treatment, vol. 51, pp. 3970-3978, 2013.

[19] K. Gao, X. Xi, Z. Wang, Y. Ma, S. Chen, X. Ye, et al., "Use of support vector machine model to predict membrane permeate flux," Desalination and Water Treatment, vol. 57, no. 36, pp. 16810-16821, 2015.

[20] C.-W. Hsu, C.-C. Chang, and C.-J. Lin, “A practical guide to support vector classification,” pp. 1-16, 2003.

[21] K. P. Murphy, "Machine learning: a probabilistic perspective," MIT press, 2012.

[22] S. A. G. Shirazi and M. B. Menhaj, "A new genetic based algorithm for channel assignment problems," in Computational Intelligence, Theory and Applications, ed: Springer, pp. 85-91, 2006.

[23] A. Ghasempour, "Using a genetic-based algorithm to solve the scheduling optimization problem for long-range molecular communications in nanonetworks," 2015 IEEE 26th Annual International Symposium on Personal, Indoor, and Mobile Radio Communications (PIMRC), Hong Kong, pp. 1825-1829, 2015.

[24] G. McLachlan, K.-A. Do, and C. Ambroise, “Analyzing microarray gene expression data," John Wiley \& Sons, vol. 422, 2005

[25] E. L. Lehmann and G. Casella, “Theory of point estimation,” Springer Science \& Business Media, 2006. 